\title{
Influência das variáveis climáticas na produção florestal via modelo 3-PG no Norte de Minas Gerais
}

\author{
Influence of climate variables in forest production via \\ 3-PG model in the northern of Minas Gerais State
}

\author{
Carlos Magno Moreira de Oliveira1, Rafael Coll Delgado², Emanuel José Gomes de Araújoº, \\ André Quintão de Almeida ${ }^{4}$, Thais Cristian Rosa ${ }^{5}$ e José Francisco de Oliveira Júnior ${ }^{2}$
}

\begin{abstract}
Resumo
Este trabalho teve por objetivo avaliar a influência das variáveis climáticas sobre a produção de plantios de Eucalyptus spp. na região Norte de Minas Gerais utilizando o modelo ecofisiológico de crescimento 3-PG (Physiological Principles in Predicting Growth). O 3-PG foi executado com base em médias históricas e mensais obtidas pelo INMET (Instituto Nacional de Meteorologia) e satélite TRMM (Tropical Rainfall Measuring Mission). A correlação linear (Pearson) ( $r$ ), coeficiente de determinação $\left(R^{2}\right)$, erro padrão da estimativa $\left(S_{y x}\right)$, eficiência do modelo $(E F)$ e raiz do erro quadrático médio (RMSE) foram utilizados para avaliar as estimativas realizadas pelo modelo 3-PG. As variáveis dendrométricas, diâmetro médio a altura do peito (DAP $-\mathrm{cm}$ ), altura total $(\mathrm{m})$, volume $\left(\mathrm{m}^{3} \cdot \mathrm{ha}^{-1}\right)$ e incremento médio anual (IMA $-\mathrm{m}^{3}$.ha-1), estimadas pelo 3-PG, com média histórica, apresentaram alta variação, com tendência de subestimativa das variáveis. As estatísticas $r$ e $R^{2}$ apresentaram valores satisfatórios $(>0,60)$. Porém observou-se que o erro das estimativas tende a ser maior quando se utiliza média histórica. O modelo teve redução da EF de 0,58 para 0,50 para o IMA. Durante janeiro de 2005 a dezembro de 2011 não houve redução da produção, caso as variáveis climáticas tivessem comportamento semelhante às médias históricas. Dentre os moduladores ambientais, observou-se que o $f A S$ teve maior restrição sobre o crescimento das árvores nessa região, seguido do modulador $f D P V$, o que pode estar relacionado às altas temperaturas encontradas na região. A utilização de normais climatológicas mostrou-se adequada para analisar o potencial produtivo de novas áreas para produção de Eucalyptus spp. na região, no entanto deve-se atentar para possíveis variações climáticas que podem reduzir a produção.
\end{abstract}

Palavras-chave: efeitos climáticos; modelagem ecofisiológica; variáveis dendrométricas.

\begin{abstract}
This study aimed to evaluate the influence of climate variables on the production of Eucalyptus spp. plantations in northern Minas Gerais State using the eco-physiological growth model 3-PG (Physiological Principles in Predicting Growth). The 3-PG was performed based on historical and monthly averages by INMET (Instituto Nacional de Meteorologia) and satellite TRMM (Tropical Rainfall Measuring Mission). The linear correlation (Pearson) $(r)$, the coefficient of determination $\left(R^{2}\right)$, standard error of estimate $\left(S_{y x}\right)$, model efficiency (EF) and root mean square error (RMSE) were used to evaluate the estimates produced by the model 3-PG. The dendrometric variables, mean diameter at breast height ( $\mathrm{DBH}-\mathrm{cm})$, total height $(\mathrm{m})$, volume $\left(\mathrm{m}^{3} \cdot \mathrm{ha}^{-1}\right)$ and mean annual increment $\left(\mathrm{MAl}-\mathrm{m}^{3}\right.$.ha- $\left.{ }^{-1}\right)$ estimated by the 3-PG, through historical average showed high variation, with a trend of underestimation of the variables. The $r$ statistics and $R^{2}$ showed satisfactory values $(>0.60)$. However, it was observed that the error of the estimate tends to be higher when using historical means. The model had reduced EF of 0.58 to 0.50 for the MAI. During
\end{abstract}

1Professor. IFMG - Instituto Federal do Norte de Minas Gerais. Campus Arinos. Rodovia MG 202, km 407 - Arinos-Buritis - 38680000 - Caixa postal 05 - Arinos, MG, Brasil. E-mail: carlos.moreira@ifnmg.edu.br

2Professor Adjunto no Departamento de Ciências Ambientais. UFRRJ - Universidade Federal Rural do Rio de Janeiro / Instituto de Florestas. Rodovia BR 465, km 07 - 23890000 - Seropédica, RJ, Brasil. E-mail: rafaelcolldelgado32@gmail. com; junior inpe@hotmail.com

3Professor Adjunto no Departamento de Silvicultura. UFRRJ - Universidade Federal Rural do Rio de Janeiro / Instituto de Florestas, Departamento de Silvicultura. Rodovia BR 465, km 07 - 23890000 - Seropédica, RJ, Brasil. E-mail: ejgaraujo@gmail.com.

4Professor Adjunto do Departamento de Engenharia Agrícola. UFS - Universidade Federal de Sergipe / Centro de Ciências Agrárias Aplicadas. Av. Marechal Rondon, s/n - Jardim Rosa Elze - 49100000 - São Cristóvão, SE, Brasil. E-mail: andre.almeida@ufs.br

5Engenheira Florestal. Vallourec Florestal Ltda. Rua Honduras, 78 - Bairro Léo Batista - 35790-000 - Curvelo, MG, Brasil. E-mail: thais.rosa@vallourec.com 
January 2005 to December 2011 there was no reduction in production if climatic variables had similar behavior to historical means. Among the environmental modulators, it was observed that $f A S$ had a greater restriction on the growth of trees in this region, followed by the $f D P V$ modulator, which may associated the high temperatures encountered in the region. The use of normal climatological situations proved to be appropriate to analyze the productive potential of new areas for production Eucalyptus spp. in the region, however attention must be paid to possible climatic variations that may reduce production.

Keywords: climatic effects; ecophysiological modeling; dendrometric variables.

\section{INTRODUÇÃO}

Mudanças no comportamento das variáveis climáticas (temperatura do ar e chuva) tem influenciado diretamente na produção das florestas plantadas e, por conseguinte, o abastecimento industrial (BAESSO et al., 2010). A partir dessas mudanças no clima há a necessidade de entender a relação entre as mudanças climáticas e o seu impacto nas florestas, o que auxilia no manejo florestal (COOPS; WARING, 2011) e no aumento substancial de incêndios florestais (CAÚLA et al., 2015). Estudos demonstram que há forte relação entre o crescimento e a disponibilidade de água (STAPE et. al., 2004; SOUZA et al., 2006; STAPE et al., 2010; COOPS et al., 2012), sendo que longos períodos de estiagem e severidade de seca podem reduzir a produtividade dos plantios de eucaliptos, ocasionando na mortalidade de alguns indivíduos (ALMEIDA et al., 2010).

A redução da chuva pode ocasionar em deficiências na formação dos indivíduos florestais e redução no incremento do plantio (BUGG et al., 2006), tornando-se necessário compreender os efeitos de fatores climáticos sobre a eficiência no uso da água pelas árvores (ALMEIDA et al., 2007). Alguns modelos relacionam variáveis climáticas ao desenvolvimento de algumas espécies, por vezes, incluindo o relevo, propriedades do solo e distribuição da chuva. A relação dessas variáveis com a produção florestal pode ser analisada por meio de modelos estatísticos e matemáticos tais como regressão linear múltipla (RLM), redes neurais artificiais (RNAs), análise harmônica e espectral (AHE) e árvore de decisão (AD), sendo possível indicar a espécie que melhor se adaptada às condições climáticas (BUGG et al., 2006; CAÚlA et al., 2015; COOPS; WARING, 2011; COSENZA et al., 2015; DELGADO et al., 2012).

Os modelos baseados em processos (MBP) ou modelos ecofisiológicos possuem algumas vantagens quando comparados aos modelos empíricos para previsão de cenários de produção florestal. Os métodos estatísticos convencionais de crescimento e produção baseiam-se em dados dendrométricos históricos do povoamento, limitando-se as predições de crescimento futuro por desconsiderar as variações climáticas (LU et al., 2015). Além disso, os MBP consideram variações sazonais das variáveis meteorológicas, por exemplo, a radiação solar global, umidade relativa do ar e temperatura do ar, propriedades relativas ao solo, assim como tratos culturais aplicados ao povoamento que podem influenciar na produção fotossintética e no crescimento da árvore na porção acima e abaixo do solo (COOPS; WARING, 2011; ZHAO et al., 2009). A partir dos modelos baseados em processos podem-se avaliar quais variáveis ambientais tem maior restrição sobre o crescimento em função das variações climáticas (BAESSO et al., 2010; PINKARD; BATTAGLIA, 2001; STAPE, et al., 2004), porém é necessário conhecimento detalhado sobre as características fisiológicas da espécie de interesse (COOPS; WARING, 2011). Nessa perspectiva, o modelo 3-PG (Physiological Principles in Predicting Growth) tem sido muito empregado para estimativa de produção florestal frente às variações climáticas (ALMEIDA et al., 2007, 2004a; GONZÁLEZ-GARCÍA et al., 2016; STAPE et al., 2004).

Baseado no exposto, os objetivos do trabalho são: i) avaliar a influência das variáveis climáticas sobre o desenvolvimento de Eucalyptus spp. na região Norte de Minas Gerais utilizando o modelo 3-PG com base nas médias históricas e médias mensais de dados climáticos para estimar as variáveis dendrométricas e ii) avaliar os efeitos do déficit de pressão de vapor d'água e a disponibilidade de água no solo sobre o crescimento do povoamento.

\section{MATERIAL E MÉTODOS}

\section{Área de Estudo}

A área de estudo está localizada no município de Bocaiúva, no Norte do estado de Minas Gerais (Figura 1). Trata-se de 313 hectares de plantios comerciais com a espécie de Eucalyptus spp. no espa- 
çamento 3 x 3 m. O clima da região, segundo a classificação de Köppen, é do tipo "Aw" tropical de savana, com inverno seco e verão chuvoso, com temperatura média anual de $24,1^{\circ} \mathrm{C}$ e chuva média anual entre $1.000 \mathrm{~mm}$ a $1.100 \mathrm{~mm}$ (DURÃES et al., 2014).
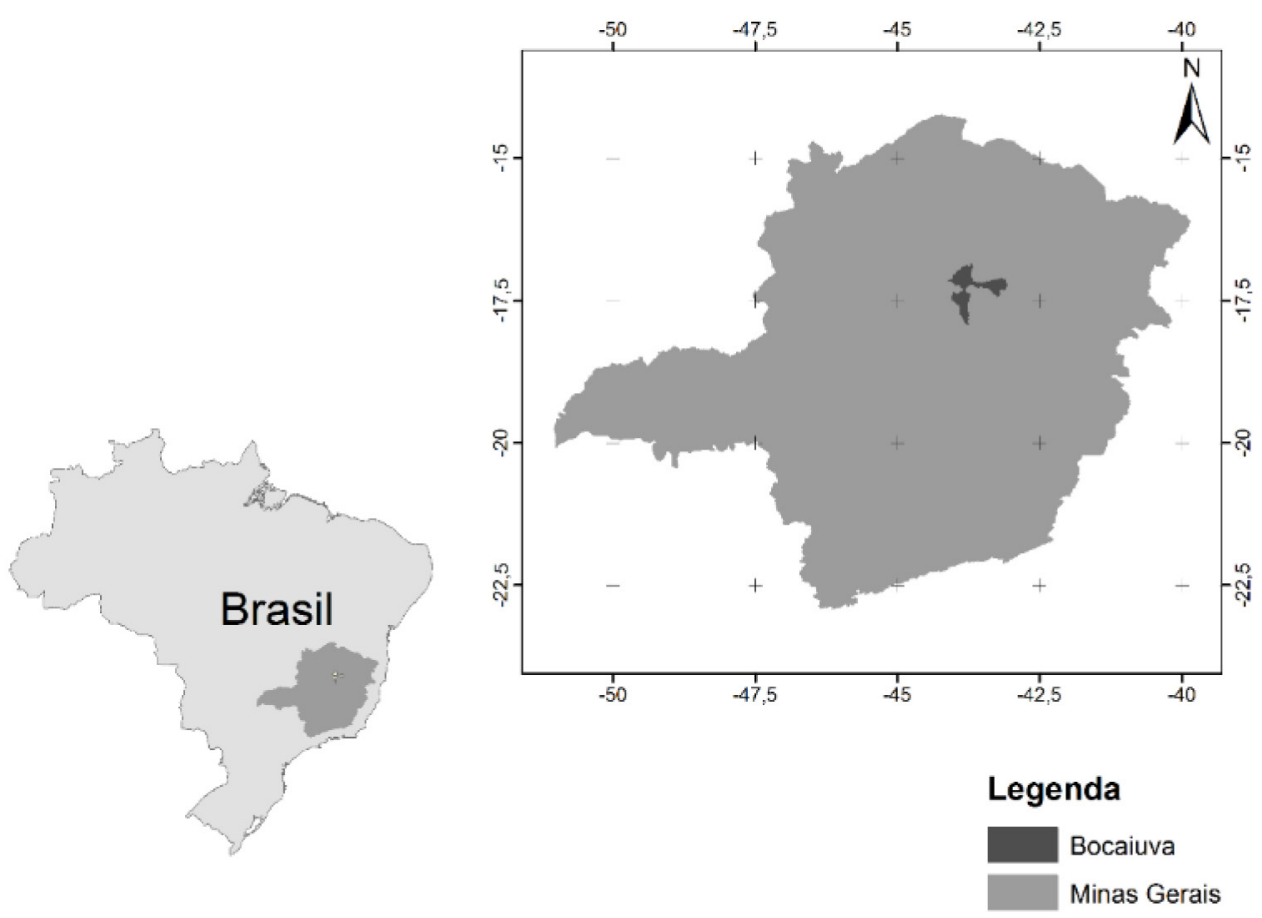

Figura 1. Localização do município de Bocaiúva (MG).

Figure 1. Location of the county of Bocaiúva (MG).

\section{Parâmetros do modelo 3-PG}

O 3-PG é controlado por fatores que restringem a abertura dos estômatos, como a limitação do déficit de pressão de vapor d'água (DPV) e disponibilidade de água no solo (AS). O modificador déficit de pressão de vapor d'água $(f D P V)$ é negativamente e exponencialmente relacionado com a média mensal do déficit de pressão de vapor d'água do ar por meio do coeficiente de resposta estomática ao déficit de pressão de vapor d'água $\left(\mathrm{K}_{\mathrm{g}}\right)$. O balanço de água no solo é a diferença entre a chuva e a evapotranspiração, sendo que a água é drenada quando a capacidade de retenção de água é excedida na zona de enraizamento. O $f A S$ é o modificador que regula a disponibilidade de água no solo e está inversamente relacionado com a disponibilidade de água no solo e depende da textura do solo (STAPE et al., 2004). Além desses, o modelo considera modificadores relativos a temperatura do ar, geada, balanço de água e nutrição da planta (LANDSBERG et al., 2003).

O modelo necessita de informações climáticas e edáficas, como disponibilidade de água no solo na zona das raízes, número de indivíduos por hectare, biomassa total alocado nas raízes, fuste e parte aérea e a razão de fertilidade do solo (LANDSBERG; WARING, 1997; SANDS; LANDSBERG, 2002). Também são necessários parâmetros específicos da espécie, que podem ser obtidos por meio de parametrização ou pela literatura (LANDSBERG et al., 2003).

Foi utilizada a parametrização proposta por Borges et al. (2012) para o cerrado em Minas Gerais, com alteração dos parâmetros das equações alométricas relativas ao volume e altura; do parâmetro fullCanAge relacionado à idade de fechamento do dossel, ou seja, quando se tem a máxima interceptação da radiação fotossinteticamente ativa (RFA) pelas folhas; e StemPower (nS) que relaciona a biomassa do stem (tronco + galhos + casca) e o diâmetro a altura do peito a 1,30 m do solo (DAP, $\mathrm{cm})$. Por meio de parametrizações, foram obtidos novos parâmetros para os modelos alométricos relativos à altura $(\mathrm{m})$ e volume $\left(\mathrm{m}^{3} \cdot \mathrm{ha}^{-1}\right)$, a partir de 2030 observações de dados de altura, volume e DAP, provenientes de inventário florestal contínuo, segundo as equações abaixo:

$$
\begin{aligned}
& H=0,60 \cdot D A P^{1,33} \\
& V=0,23 \cdot D A P^{2,47}
\end{aligned}
$$


Dessa forma, os parâmetros propostos por Borges et al. (2012) em relação a constante de relacionamento entre altura e DAP $(\mathrm{aH})$, potência de relacionamento entre altura total e DAP (nHB), constante de relacionamento entre volume e DAP $(\mathrm{aV})$, potência de relacionamento entre volume e DAP $(\mathrm{nVB})$ foram alterados para 0,60, 1,33, 0,23 e 2,47, respectivamente. Foram atribuídos os valores 2 e 3,22 aos parâmetros fullCanAge e StemPower, respectivamente, por apresentarem melhores resultados durante o ajuste do modelo. Para estimativa das variáveis foi utilizado a versão 2.4 do 3-PG.

\section{Dados climáticos}

A influência das variáveis climáticas sobre a produção florestal via o 3-PG foi avaliada utilizando dados nas escalas mensal (entre os meses de 2005 e 2011) e histórica. A série temporal mensal contém informações da temperatura mínima e máxima do $\operatorname{ar}\left(\mathrm{t}_{\mathrm{n}}\right.$ e $\left.\mathrm{t}_{\mathrm{x}^{\prime}}{ }^{\circ} \mathrm{C}\right)$, radiação solar global $\left(\mathrm{R}_{\mathrm{s}^{\prime}}\right.$ MJ.m. ${ }^{-2} \cdot$ dia $\left.^{-1}\right)$ e chuva ( $\mathrm{mm}$ ). Dados da $\mathrm{t}_{\mathrm{x}}$ e $\mathrm{t}_{\mathrm{n}}$ foram obtidos de Estações Meteorológicas Automáticas (EMA) do Instituto Nacional de Meteorologia (INMET), a chuva foi obtida do satélite tropical TRMM (Tropical Rainfall Measuring Mission) via produto 3B43, com resolução espacial de $20 \mathrm{~km} \mathrm{e}$ informações de chuva horária que foram convertidos para média mensal (OLIVEIRA JÚNIOR et al., 2014) (Figura 2). Segundo Almeida et al. (2015) os dados do produto 3B43 podem ser utilizados quando não há dados de Estações Meteorológicas Convencionais (EMC), com boa precisão e uma malha densa de rede de estações. As informações de $\mathrm{R}_{\mathrm{s}}$ foram obtidas pelo método proposto pela FAO 56 (Food and Agriculture Organization of the United Nations) que requer informações de latitude $\left({ }^{\circ}\right)$ do local e número de horas de brilho solar por dia (n), sendo este último foi obtido da base de dados do INMET (ALLEN et al., 1998).

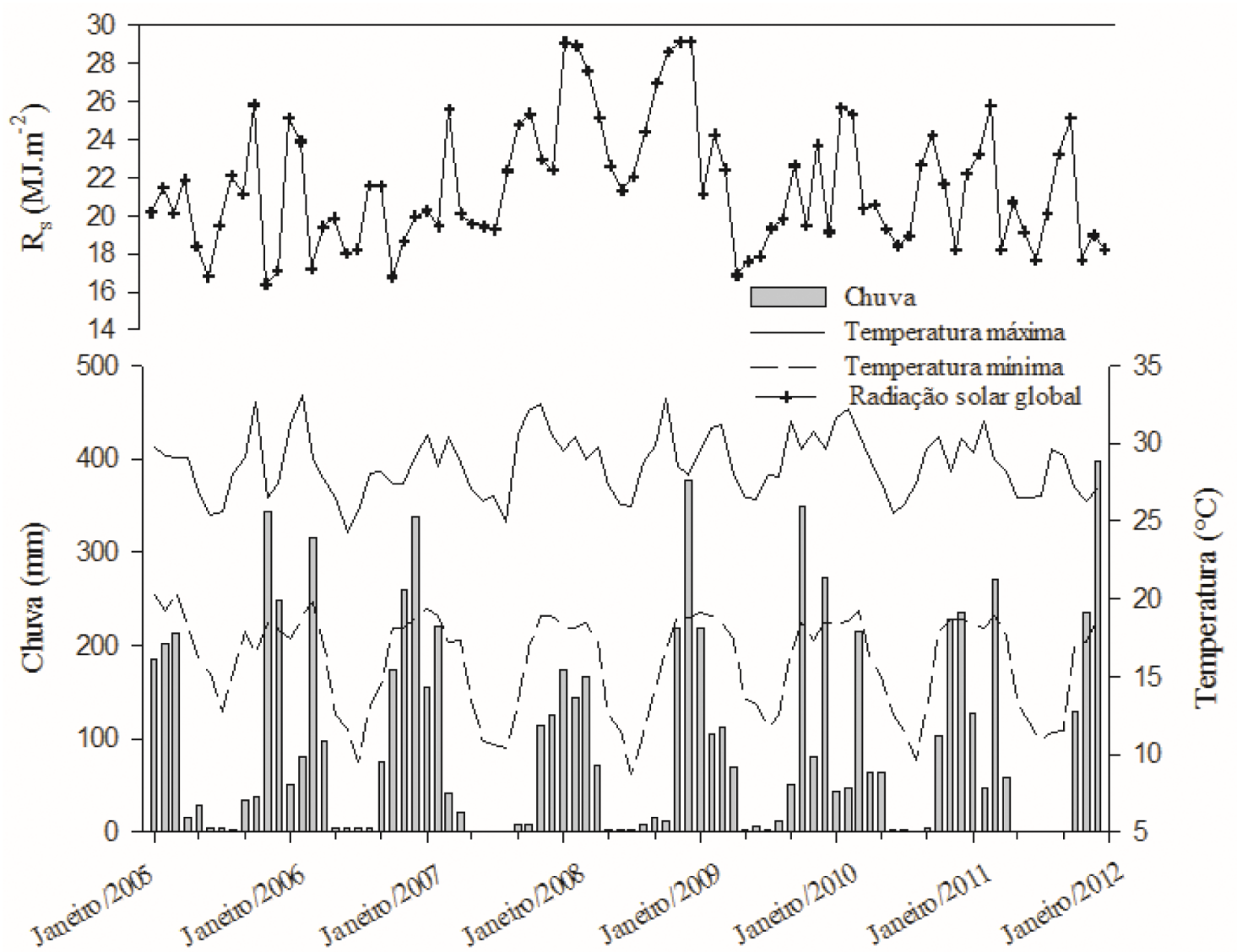

Figura 2. Média mensal de chuva $(\mathrm{mm})$, temperatura máxima $\left(\mathrm{t}_{\mathrm{x}},{ }^{\circ} \mathrm{C}\right)$ e temperatura mínima do ar $\left(\mathrm{t}_{\mathrm{n}},{ }^{\circ} \mathrm{C}\right)$ e radiação solar global (Rs, MJ.m²) para a região de Janeiro de 2005 a Dezembro de 2011.

Figure 2. Mean monthly rainfall $(\mathrm{mm})$, maximum temperature $\left(t_{x},{ }^{\circ} \mathrm{C}\right)$ and minimum air temperature $\left(t_{n^{\prime}}{ }^{\circ} \mathrm{C}\right)$ and global solar radiation (Rs, MJ.m²) to the region from January 2005 to December 2011.

A série temporal de média histórica é composta pela $\mathrm{R}_{\mathrm{s}}$ média anual obtida do modelo GL1.2 (GLobal) que estima a radiação solar global por meio de imagens do satélite GOES (Geostationary Operational Environmental Satélites) que podem ser obtidas no seguinte endereço eletrônico: http://satelite. cptec.inpe.br/radiacao. O modelo GL 1.2 trabalha com informações das bandas do visível (VIS) e in- 
fravermelho (IV), isso resulta em médias diárias de radiação solar global sobre grade de $0,4^{\circ} \times 0,4^{\circ}$ e médias subsequentes em 5 dias (pêntadas) no período de 26 de dezembro de 1995 a 24 de dezembro de 2006, resultando em 803 pêntadas. Os dados foram processados no programa OpenGrADS 2.0, obtendo-se a média mensal $\mathrm{R}_{\mathrm{s}}\left(\mathrm{MJ} \cdot \mathrm{m}^{-2}\right.$. êe $^{-1}$ ) no período de 10 anos; dados de chuva, $\mathrm{t}_{\mathrm{n}} \mathrm{e} \mathrm{t}_{\mathrm{x}}$ foram obtidos pelas normais climatológicas do Brasil disponibilizado pelo INMET. Em Bocaiúva não possui normais climatológicas, por isso foram utilizados dados do município de Curvelo (MG) (Figura 3).

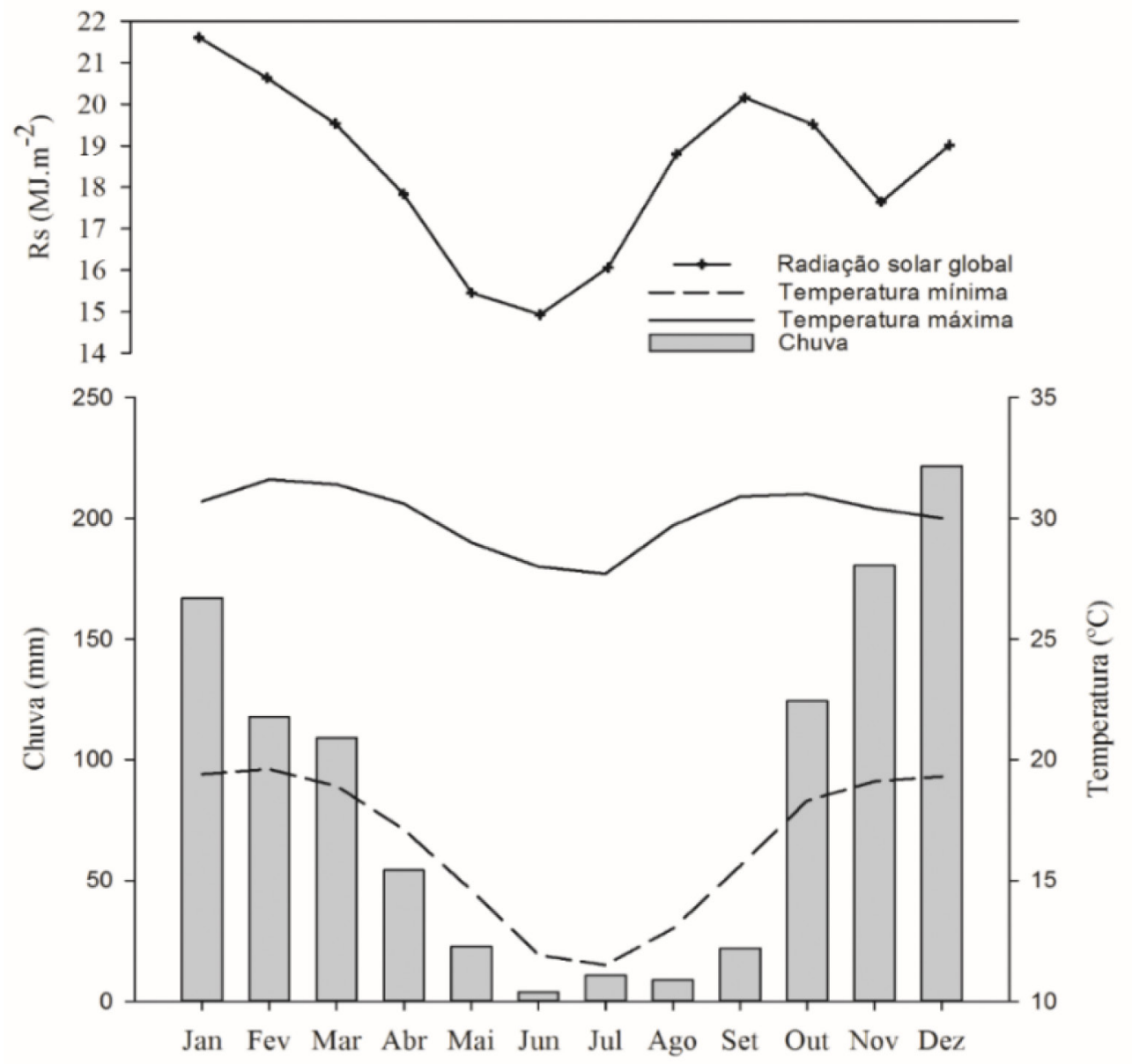

Figura 3. Média histórica de chuva $(\mathrm{mm})$, temperatura máxima $\left(\mathrm{t}_{x^{\prime}},{ }^{\circ} \mathrm{C}\right)$ e temperatura mínima do ar $\left(\mathrm{t}_{\mathrm{n}},{ }^{\circ} \mathrm{C}\right)$ e radiação solar global (Rs, MJ.m-2) para a região.

Figure 3. Historical mean rainfall $(\mathrm{mm})$, maximum temperature $\left(t_{x^{\prime}}{ }^{\circ} \mathrm{C}\right)$ and minimum air temperature $\left(t_{n},{ }^{\circ} \mathrm{C}\right)$ and global solar radiation ( $\mathrm{Rs}, \mathrm{MJ} . \mathrm{m}^{-2}$ ) to the region.

Outras informações necessárias para executar o 3-PG são latitude do local do plantio, fertilidade, textura do solo e capacidade mínima e máxima de retenção de água no solo. A variável referente a fertilidade do solo (FR) varia de 0 a 1 , sendo que 1 indica que há alta fertilidade, sem restrições sobre o crescimento do plantio, e 0, quando há redução da fertilidade. Dessa forma, foi utilizado o valor 1 para a FR, pois foi realizada adubação de base, atendendo as necessidades da cultura. A textura do solo foi obtida a partir do levantamento realizado em campo, sendo constatada a predominância de solo tipo latossolo vermelho com textura argilosa. Segundo Borges et al. (2012) nessa região há uma variação da disponibilidade de água no solo (DAS) de $200 \mathrm{~mm}$ a $260 \mathrm{~mm}$ na profundidade efetiva do sistema radicular de aproximadamente $2,0 \mathrm{~m}$.

\section{Validação do modelo 3-PG}

O 3-PG foi configurado para gerar estimativas mensais do primeiro ao sétimo ano do povoamento. A acurácia das estimativas do 3-PG com base em médias mensais e normais climatológicas foi realizada por meio de 335 medições do inventário florestal contínuo no período de 7 anos, coletados em 10 talhões, e sobreposto aos valores médios estimados pelo modelo com o intuito de avaliar a eficiência na estimativa das variáveis dendométricas: diâmetro a 1,30 m do solo (DAP - cm), altura 
total $(\mathrm{m})$, volume $\left(\mathrm{m}^{3} \cdot \mathrm{ha}^{-1}\right)$ e incremento médio anual (IMA - $\left.\mathrm{m}^{3} \cdot \mathrm{ha}^{-1} \cdot \mathrm{ano}^{-1}\right)$. Para comparação dos dados estimados com observados, as medições do inventário florestal foram agrupadas nas diferentes idades do povoamento de forma que coincidissem com as idades de saída do modelo 3-PG, com um total de 25 observações médias. A partir dessas informações, foram aplicados os seguintes métodos estatísticos para as variáveis DAP, altura total, volume e IMA: correlação linear (Pearson) $(\mathrm{r})$, coeficiente de determinação $\left(\mathrm{R}^{2}\right)$, erro padrão da estimativa $\left(\mathrm{S}_{\mathrm{yx}}\right)$, erro padrão da estimativa em porcentagem $\left(\mathrm{S}_{\mathrm{yx}} \%\right)$, eficiência do modelo (EF) e raiz do erro quadrático médio (RMSE). As equações utilizadas seguem abaixo:

$$
\begin{aligned}
& r=\frac{\sum O_{i} P_{i}}{\sqrt{\sum O_{i}^{2} \sqrt{\sum P_{i}^{2}}}} \\
& R^{2}=1-\left(\frac{S Q_{r e s}}{S Q_{t}}\right) \\
& S_{y x}=\sqrt{\frac{\sum_{i=1}^{n}\left(P_{i}-O_{i}\right)^{2}}{n-p-1}} \quad \therefore S_{y x} \%=\frac{S_{y x}}{\sigma} * 100 \\
& E F=1-\frac{\sum\left(O_{i}-P_{i}\right)^{2}}{\sum\left(O_{i}-\bar{O}\right)^{2}} \\
& R M S E=\sqrt{\sum_{i=1}^{n} \frac{\left(P_{i}-O_{i}\right)^{2}}{n}}
\end{aligned}
$$

em que, $\mathrm{P}_{\mathrm{i}}$ é o iésimo valor estimado pelo modelo 3-PG, $\mathrm{O}_{\mathrm{i}}$ é o iésimo valor observado no inventário florestal, $\overline{\mathrm{O}}$ é a média aritmética da variável observada, $\mathrm{SQ}_{\mathrm{res}}$ é a soma dos quadrados dos resíduos, $\mathrm{SQ}_{\mathrm{t}}$ é a soma total dos quadrados, n é o número de dados observados.

\section{RESULTADOS E DISCUSSÃO}

A modelagem realizada pelo 3-PG apresentou resultados similares aos observados no inventário florestal nos primeiros 4 anos do povoamento de Eucalyptus spp. (Figura 4). Segundo Almeida et al. (2004b), o efeito de secas severas e estiagens prolongadas podem ser captadas pelo 3-PG quando utiliza-se médias mensais atuais em comparação as estimativas obtidas por médias históricas. Os resultados obtidos baseados em médias mensais mostraram que, caso o regime climático atual fosse semelhante as normais climatológicas, não haveria perda na produção nos primeiros 4 anos do povoamento de Eucalyptus spp., ou seja, a produção alcançada seria muito próxima a obtida atualmente. Após os 4 anos, as condições climáticas atuais favoreceram o desenvolvimento do povoamento, o que pode estar relacionado a diminuição da restrição de algum modificador ambiental do modelo 3-PG (Figura 4).

Observa-se tendência de subestimativa das variáveis dendrométricas ao longo do tempo quando se utiliza as normais climatológicas como dado de entrada no modelo 3-PG, visto que a linha de tendência entre o observado e estimado ficou acima da linha 1:1 (Figura 4-b,d,f,h). Após o quarto ano, o modelo tende a subestimar as variáveis $\mathrm{H}$ e DAP e, consequentemente, se reflete nas variáveis volume e IMA, pois são dependentes do DAP e H (Figura 4-a,c,e,g). Os efeitos da chuva podem variar de acordo com a idade do plantio e também com os períodos de seca prolongada, isso demonstra que médias históricas são pouco eficientes para captar essas variações no crescimento (ALMEIDA et al., 2004b). Outro fato reside na própria variabilidade espacial e temporal da chuva em uma região, que por sua vez dependem dos sistemas meteorológicos que nela atuam e dos modos de variabilidade climática (El Niño-Oscilação Sul - ENOS e Oscilação Decadal do Pacífico - ODP) que interferem nos sistemas, que ora aumentam a produção e ora contribuem para inibição da chuva (LYRA et al., 2017; OLIVEIRA JÚNIOR et al., 2014). 
A estatística aplicada as variáveis dendrométricas mostrou que o coeficiente $\mathrm{r}$ apresentou forte correlação positiva para o DAP, $\mathrm{H}$ e V $(>0,90)$ (Tabela 1$)$. O coeficiente $\mathrm{R}^{2}$ para médias mensais mostrou-se muito semelhante ao $\mathrm{R}^{2}$ para as variáveis dendrométricas estimadas utilizando-se média histórica, isso corrobora com os resultados de Borges et al. (2012), onde foram utilizadas médias históricas para realizar as estimativas via modelo 3-PG, sendo que para altura, DAP e volume o ${ }^{2}$ foi de 0,95, 0,97 e 0,92, respectivamente.

$\mathrm{O} \mathrm{R}^{2}$ relativo ao IMA estimado com médias mensais e normais climatológicas ficou abaixo do encontrado por Almeida et al. (2004b), próximo a 0,90, porém o pico do IMA foi próximo aos 6 anos indo ao encontro dos valores encontrados por esses autores para regiões ao sul da Bahia e ao Norte do Espírito Santo (Tabela 1). Nesse estudo foram utilizados dados de várias espécies de Eucalyptus o que pode contribuir para redução dos valores do $\mathrm{R}^{2}$ e aumentar o $\mathrm{S}_{\mathrm{yx}}$ para o IMA, quando comparado ao encontrado por Almeida et al. (2004b) que utilizaram dados de Eucatyptus grandis para validar o 3-PG. Os valores de $\mathrm{S}_{\mathrm{xy}}(\%)$ e o RMSE aumentaram quando utilizado as normais climatológicas, com isso ocorreu uma diferença entre o observado e estimado. A EF apresentou pouca diferença para as variáveis dendrométricas estimadas com os dados climáticos nas duas escalas. A menor eficiência do modelo foi para o IMA que apresentou EF de 0,58, estimado por meio de médias mensais (Tabela 1).

Tabela 1. Resumo das análises estatísticas das variáveis dendométricas DAP $(\mathrm{cm})$, altura $(\mathrm{m})$, volume $\left(\mathrm{m}^{3} \cdot h \mathrm{ha}^{-1}\right)$ e IMA ( $\mathrm{m}^{3} \cdot \mathrm{ha}^{-1}$.ano ${ }^{-1}$ ) estimadas por meio de médias mensais (mensal) e normais climatológicas (NC) para município de Bocaiúva (MG).

Table 1. Summary of statistical analysis of the variables dendometric DBH $(\mathrm{cm})$, height $(\mathrm{m})$, volume $\left(\mathrm{m}^{3} . h \mathrm{~h}^{-1}\right)$ and IMA ( $\mathrm{m}^{3}$.ha $\mathrm{h}^{-1}$. ano-1) estimated using monthly averages (monthly) and climatological normal (NC) to the county of Bocaiúva (MG).

\begin{tabular}{|c|c|c|c|c|c|c|c|}
\hline Variáveis & $\mathbf{N}$ & $\mathbf{r}$ & $\overline{\mathbf{R}^{2}}$ & $\mathbf{S}_{u x}$ & $\overline{S_{v y}(\%)}$ & $\overline{E F}$ & RMSE \\
\hline DAP $(\mathrm{cm})-$ mensal & 25 & 0,97 & 0,94 & 1,24 & 10,74 & 0,87 & 1,19 \\
\hline $\mathrm{DAP}(\mathrm{cm})-\mathrm{NC}$ & 25 & 0,96 & 0,93 & 1,51 & 13,09 & 0,81 & 1,45 \\
\hline $\mathrm{H}(\mathrm{m})$ - mensal & 25 & 0,92 & 0,84 & 2,76 & 17,59 & 0,80 & 2,64 \\
\hline $\mathrm{H}(\mathrm{m})-\mathrm{NC}$ & 25 & 0,91 & 0,84 & 3,00 & 19,17 & 0,76 & 2,88 \\
\hline$V\left(m^{3} \cdot h a^{-1}\right)-$ mensal & 25 & 0,92 & 0,85 & 27,11 & 26,52 & 0,84 & 26,00 \\
\hline$V\left(m^{3} \cdot h a^{-1}\right)-N C$ & 25 & 0,93 & 0,86 & 28,54 & 27,92 & 0,82 & 27,37 \\
\hline IMA $\left(\mathrm{m}^{3} \cdot \mathrm{ha}^{-1} \cdot \mathrm{ano}^{-1}\right)$ - mensal & 25 & 0,78 & 0,62 & 6,07 & 28,00 & 0,58 & 5,82 \\
\hline IMA $\left(\mathrm{m}^{3} \cdot \mathrm{ha}^{-1} \cdot \mathrm{ano}^{-1}\right)-\mathrm{NC}$ & 25 & 0,77 & 0,60 & 6,64 & 30,66 & 0,50 & 6,37 \\
\hline
\end{tabular}

Segundo Almeida et al. (2004b), a média histórica pode ser utilizada para avaliar o potencial produtivo de novas áreas, para implantação de povoamento florestais. Porém, pode incorrer em estimativas pouco precisas para tomada de decisão, devendo-se considerar os efeitos da variabilidade climática sobre cenários de produção florestal. Além disso, o uso de médias históricas pode acarretar em superestimativas de até 25\% (ALMEIDA et al., 2010). Diferente do que foi observado por esses autores, nesse estudo observou-se que a média histórica subestimou o volume médio ao final da rotação em aproximadamente $15 \%$.

A temperatura média ficou abaixo da temperatura ótima $\left(<25^{\circ} \mathrm{C}\right)$ de abril a setembro, para as normais climatológicas, o que pode reduzir o desempenho fisiológico das plantas, já que o modelo considera a temperatura do ar como uma das variáveis que regulam a atividade fotossintética por meio do modificador $f T$. Resultados semelhantes foram encontrados por Coops e Waring (2011) com Pinus contorta Dougl. no Noroeste (NW) da América do Norte (AN). Zhao et al. (2009) demonstrou, por meio de cenários de produção, que a variação da temperatura ótima $\left(\mathrm{T}_{\mathrm{opt}}\right)$ de crescimento no cultivo de Chinese fir resulta em diferentes níveis de produção florestal. Segundo os autores, o aumento progressivo da $\mathrm{T}_{\mathrm{opt}}$ de $0-2^{\circ} \mathrm{C}$ no modelo 3-PG, resultou em estimativas de produção elevado, reduzindo o período de rotação do plantio. Porém, ao aumentar a $\mathrm{T}_{\mathrm{opt}}$ em mais de $2{ }^{\circ} \mathrm{C}$ pode resultar em perdas na produção, devido o fechamento dos estômatos (ZHAO et al., 2009; HUNG et al., 2016). Como a região de estudo tem por característica altas temperaturas do ar durante o ano (DELGADO et al., 2012), isso pode favorecer a produção, desde que as demais variáveis ambientais, que regem o desenvolvimento dos indivíduos, estejam adequadas.

Waring et al. (2014) observaram uma tendência de aumento da temperatura do ar no oeste da América do Norte, que ocasionou a redução do número de dias de geada, podendo está relacionada 
a mortalidade de indivíduos de pinus em floresta na Columbia Britânica. Por outro lado, Coops e Waring (2011) mencionam que a redução do período de inverno, pode favorecer o período de crescimento para espécies adaptadas como Pinus e Douglas-fir na região noroeste da Columbia Britânica. Dessa forma, a região tropical pode ter mortalidade de indivíduos florestais pelo aumento demasiado da temperatura do ar em locais com estiagens prolongadas, ocasionando em estresse hídrico como sugerido por Coops e Waring (2010) ou até favorecer a produção quando não houver estresse hídrico ou restrição de outra variável, favorecendo a fotossíntese.
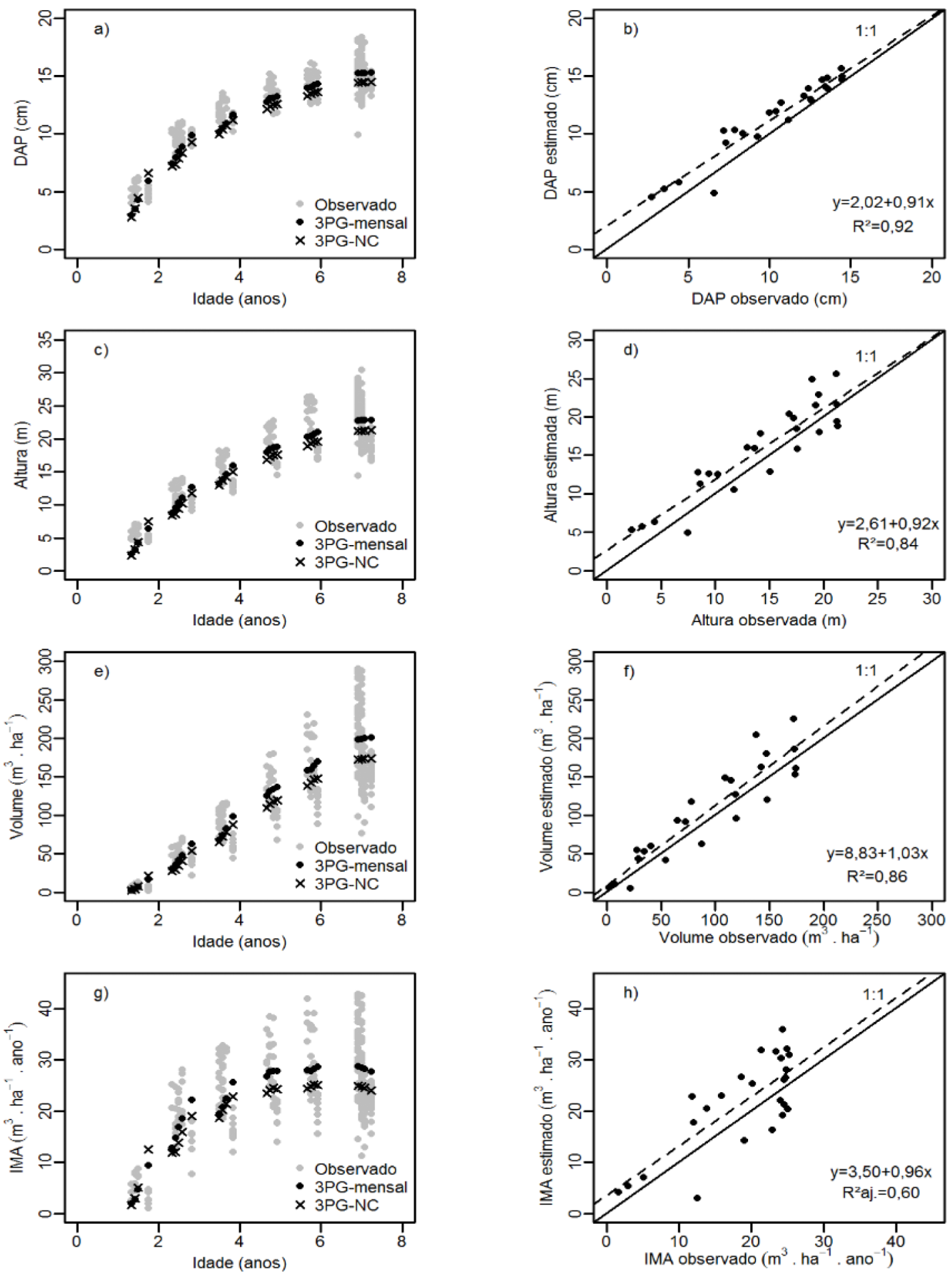

Figura 4. Distribuição das variáveis DAP (a), altura (c), volume (e) e IMA (g) em relação às idades do plantio. Relação entre dados observados e estimados pelo modelo 3-PG com base nas normais climatológicas, para DAP (b), altura (d), volume (f) e IMA (h) para o município de Bocaiúva (MG).

Figure 4. Distribution of variables DBH (a) height (c) volume (e) and IMA (g) in relation to planting ages. Relationship between observed data and estimated by the model 3-PG com based on climatological normals for DBH (b), height (d) volume (f) and IMA (h) to the county of Bocaiúva (MG). 
O modificador de disponibilidade de água no solo $(f A S)$ apresentou variação acentuada entre os meses, sendo que a partir de março a restrição sobre desenvolvimento dos indivíduos aumentou gradativamente (Figura 5a). Nos meses de julho, agosto e setembro o modificador apresentou um menor valor, ou seja, máxima restrição sobre a atividade fotossintética, mas com menor desvio padrão quando comparado aos meses de abril, maio, outubro e novembro. Após os meses de baixa pluviometria, o IMA tende a reduzir e aumentar após os meses chuvosos, demonstrando sensibilidade a variação da chuva e disponibilidade de água no solo (ALMEIDA et al., 2010; GONZÁLEZ-GARCÍA et al., 2016). Porém, torna-se necessário compreender a influência de outras variáveis relacionadas, como a profundidade do solo e o desenvolvimento das raízes (ALMEDIA et al., 2010). Os meses com menor disponibilidade de água no solo coincide com o período de menor chuva, dessa forma, a textura do solo pode auxiliar na retenção de umidade, reduzindo o estresse hídrico do plantio no período de seca e minimizando perdas na produção. Espécies adaptadas a períodos de seca prolongada são pouco afetadas pela baixa umidade no solo (GONZÁLEZ-GARCÍA et al., 2016). Por outro lado, o modificador $f D P V$ apresentou baixa variação, com valores próximos a 0,7 e com desvio padrão próximo a 0 (Figura 5a). Almeida et al. (2004b) demostraram que informações precisas de chuva e déficit de pressão de vapor d' água (DPV) tem grande influência na precisão das estimativas. Isso foi constatado pela avaliação de duas áreas, com distância de aproximadamente 50 $\mathrm{km}$, que apresentaram diferença de produção em volume de $154 \mathrm{~m}^{3} \cdot \mathrm{ha}^{-1}$ aos 7 anos.
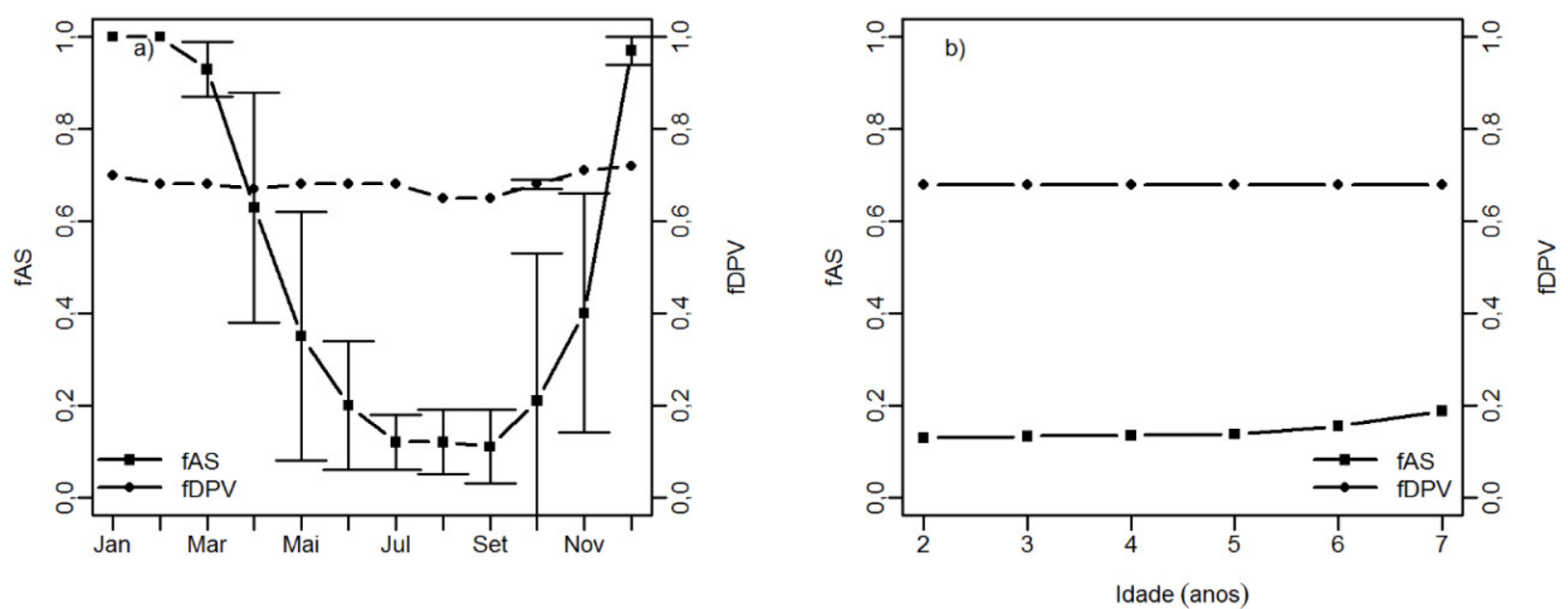

Figura 5. Variação do modificador de disponibilidade de água no solo (fAS) e modificador de déficit de pressão de vapor ( $f D P V)$ a) de Janeiro a Dezembro e b) para as idades do plantio em Bocaiúva (MG).

Figure 5. Changes in the modifier water availability in the soil ( $f A S)$ and modifier vapor pressure deficit ( $f D P V)$ a) from January to December and b) ages of stand in Bocaiúva (MG).

A Figura 5b apresenta a variação dos modificadores $f A S$ e $f D P V$ em relação as idades do plantio, com base nos dados climatológicos mensais que compreende o período em que foi realizado o inventário florestal. Observa-se que o modificador $f D P V$ permaneceu constante, entre o segundo e o sétimo ano do plantio, apresentando média de 0,68, próximo ao encontrado por Almeida et al. (2010) para plantios de eucalipto na Bahia e Espírito Santo que foi de 0,65. Como os modificadores ambientais, no 3-PG, variam de 0 a 1, sendo que 0 é alta restrição e 1 é quando não há restrição do modificador sobre o crescimento, observa-se que o $f D P V$ atuou com aproximadamente $30 \%$ de restrição sobre o desenvolvimento do plantio. Já o modificador $f A S$ apresentou restrição considerável sobre o povoamento, principalmente entre o segundo e o quinto ano (Figura 5b). Após o quinto ano, a restrição do modificador de disponibilidade de água no solo tende a reduzir, chegando a aproximadamente 0,20 no sétimo ano de produção. O período de menor restrição do $f A S$ coincide com aumento do volume e IMA no povoamento, como apresentado na Figura 3. Souza et al. (2006) observaram que em anos precedidos de estação chuvosa classificada como chuvosa e muito chuvosa na bacia do Rio Doce, o incremento periódico mensal (IPM) foi maior. Hung et al. (2016) também observou que o incremento corrente anual (ICA) pode ser maior no período chuvoso quando comparado ao período seco. Isso demonstra que se as condições climáticas atuais fossem semelhantes à média histórica, ocorreria redução no desenvolvimento das variáveis $\mathrm{DAP}, \mathrm{H}$, volume e IMA a partir dos 5 anos de idade do plantio. 
A redução da disponibilidade de água no solo e o aumento da temperatura do ar podem reduzir o índice de área foliar (IAF) e, consequentemente, redução da produção fotossintética do plantio de eucalipto, conforme observado por Battaglia et al. (1998) em povoamentos de E. nitens e E. globulus. O IAF está diretamente relacionado com a capacidade da planta em absorver radiação fotossintética, sendo que o fornecimento de água facilita o aumento da área foliar (STAPE et al., 2004). Souza et al. (2006) mencionam que em locais com maior irradiância solar e elevadas temperaturas pode aumentar a demanda evapotranspirativa do plantio, redução da condutância estomática e menor assimilação de $\mathrm{CO}_{2}$. A eficiência da condutância estomática está diretamente relacionada com o aumento da disponibilidade de água no solo e redução o déficit de pressão de vapor (ALMEIDA et al., 2007; STAPE et al., 2007). Nessa perspectiva, foi observado que o modificador $f A S$ apresentou limitação significativa sobre a atividade fotossintética em plantios de Pinus (COOPS; WARING, 2011) e em povoamentos de Acácia (HUNG et al., 2016). De acordo com Waring et al. (2014) as configurações atuais do 3-PG associadas a dados climáticos e de solo coletados em campo, ou seja, com alta precisão, permitem predizer o crescimento do dossel e a dinâmica interanual do plantio com erros próximos aos das medições de campo.

\section{CONCLUSÕES}

O uso do 3-PG com base nas normais climatológicas mostra-se adequado para estimar o potencial produtivo de novas áreas para produção florestal. Utilizando-se normais climatológicas pode incorrer em subestimativas ou superestimativas do incremento médio anual (IMA) para o período desejado, devido à baixa sensibilidade do modelo em prever variações climáticas usando dados nessa escala.

As normais climatológicas evidenciam como cada modificador ambiental age sobre o desenvolvimento do plantio para região de interesse, indicando alternativas de manejo florestal que podem reduzir possíveis perdas na produção.

As estimativas do modelo são reguladas por modificadores ambientais, sendo que nesse estudo, o $f A S$ apresentou forte restrição sobre o crescimento quando comparado ao $f D P V$, o que pode estar relacionado às altas temperaturas da região e distribuição irregular da chuva durante o ano.

\section{REFERÊNCIAS BIBLIOGRÁFICAS}

ALLEN, R.G.; JENSEN, M.E.; BORNAN, R. D. Operational estimates of reference evapotranspiration. Agronomy Journal, Madison, v.81, n.4, p.650-662, 1989.

ALMEIDA, C. T.; DELGADO, R. C.; OLIVEIRA JÚNIOR, J. F.; GOIS, G.; CAVALCANTI, A. S. Avaliação das Estimativas de Precipitação do Produto 3B43-TRMM do Estado do Amazonas. Floresta e Ambiente, Seropédica, v. 22, n. 3, p. 279-286, 2015.

ALMEIDA, A. C.; SIGGINS, A.; BATISTA, T. R.; BEADLE, C.; FONSECA, S.; LOOS, R. Mapping the effect of spatial and temporal variation in climate and soils on Eucalyptus plantation production with 3-PG, a processbased growth model. Forest Ecology and Management, Amsterdam, v. 259, n. 9, p. 1730-1740, 2010.

ALMEIDA, A. C.; SOARES, J. V.; LANDSBERG, J. J.; REZENDE, G. D. Growth and water balance of Eucalyptus grandis hybrid plantations in Brazil during a rotation for pulp production. Forest Ecology and Management, Amsterdam, v. 251, n. 1-2, p. 10-21, 2007.

ALMEIDA, A. C.; LANDSBERG, J. J.; SANDS, P. J. Parameterisation of 3-PG model for fast-growing Eucalyptus grandis plantation. Forest Ecology and Management, Amsterdam, v. 193, n. 1-2, p. 179-195, $2004 \mathrm{a}$.

ALMEIDA, A. C.; LANDSBERG, J. J.; SANDS, P. J.; AMBROGI, M. S.; FONSECA, S.; BARDDAL, S. M.; BERTOLUCCI, F. L. Needs and opportunities for using a process-based productivity model as a practical tool in Eucalyptus plantations. Forest Ecology and Management, Amsterdam, v. 193, n. 2-3, p. 167-177, 2004b. 
BAESSO, R. C. E.; RIBEIRO, A.; SILVA, M. P. Impacto das mudanças climáticas na produtividade do eucalipto na região norte do Espírito Santo e Sul da Bahia. Ciência Florestal, Santa Maria, v. 20, n. 2, p. 335-344, 2010.

BATTAGLIA, M.; CHERRY, M. L.; BEADLE, C. L.; SANDS, P. J.; HINGSTON, A. Prediction of leaf area index in eucalypt plantations: effects of water stress and temperature. Tree Physiology, Victoria, v. 18, n. 8-9, p. 521$528,1998$.

BORGES, J. S.; NEVES, J. C. L.; LOURENÇO, H. M.; BARROS, N. F.; DIAS, S. C. M. Parameterization of the 3-PG model for eucalypt in the region of cerrado in Minas Gerais state. Ciência Florestal, Santa Maria, v. 22, n. 3, p. 567-578, 2012.

BUGG, A. L.; LANDSBERG, J.; LAUGHLIN, G.; TICKLE, P.; FRAKES, I. Application of three modelling approaches to simulating tree growth in central NSW, Australia. Enviromental Modelling \& Software, Oxford, v. 21, n. 3, p. 310-319, 2006.

CAÚLA, R. H.; OLIVEIRA-JÚNIOR, J. F.; LYRA, G. B.; DELGADO, R. C.; HEILBRON FILHO, P.F.L. Overview of fire foci causes and locations in Brazil based on meteorological satellite data from 1998 to 2011. Environmental Earth Sciences, Berlim, v. 74, n. 2, p. 1497-1508, 2015.

COOPS, N. C.; WARING, R. H. A process-based approach to estimate lodgepole pine (Pinus contorta Dougl.) distribution in the Pacific Northwest under climate change. Climatic Change, Covarllis, v. 105, n. 1, p. $313-$ $328,2011$.

COOPS, N. C.; WARING, R. H.; HILKER, T. Prediction of soil properties using a process-based forest growth model to match satellite-derived estimates of leaf area index. Remote Sensing of Environment, Nova Iorque, v. 126, n.1, p. 160-173, 2012.

COSEnZA, D. N.; LEITE, H. G.; MARCATTI, G. E.; BINOTI, D. H. B.; ALCÂNTARA, A. E. M.; RODE, R. Classificação da capacidade produtiva de sítios florestais utilizando máquina de vetor de suporte e rede neural artificial. Scientia Forestalis, Piracicaba, v. 43, n. 108, p. 955-963, 2015

DELGADO, R. C.; SEDIYAMA, G. C.; COSTA, M. H ; SOARES, V. P. ; ANDRADE, R. G.Classificação espectral de área plantada com a cultura da cana-de-açúcar por meio da árvore de decisão. Engenharia Agrícola, Jaboticabal, v. 32, n. 2, p. 369-380, 2012.

DURÃES, M. C. O.; SALES, N. L. P.; NETO, S. D.; FIGUEIREDO, M. A. P. Levantamento florístico do estrato arbóreo de três fragmentos de floresta ciliar como subsídio à recomposição da vegetação do rio Cedro, Montes Claros - MG. Ciência Florestal, Santa Maria, v. 24, n. 1, p. 47-58, 2014.

GONZÁLEZ-GARCÍA, M.; ALMEIDA, A. C.; HEVIA, A.; MAJADA, J.; BEADLE, C. Application of a processbased model for predicting the productivity of Eucalyptus nitens bioenergy plantationsin Spain. Bioenergy, Nova York, v. 8, n.1, p. 194-210, 2016.

HUNG, T. T.; ALMEIDA, A. C.; EYLES, A.; MOHAMMED, C. Predicting productivity of Acacia hybrid plantations for a range of climates and soils in Vietnam. Forest Ecology and Management, Amsterdam, v. 367, n. 1, p. 97-111, 2016.

LANDSBERG, J. J.; WARING, R. H. A generalized model of forest productivity using simplified concepts of radiation-use efficiency, carbon balance and partitioning. Forest Ecology and Management, Amsterdam, v. 95, n. 3, p. 2009-228, 1997.

LANDSBERG, J. J.; WARING, R. H.; COOPS, N. C. Performance of the forest productivity model 3-PG applied to a wide range of forest types. Forest Ecology and Management, Amsterdam, v. 172, n. 2-3, p. 199-214, 2003. 
Oliveira et al. - Influência das variáveis climáticas na produção florestal via modelo 3-PG no Norte de Minas Gerais

LYRA, G. B.; OLIVEIRA-JÚNIOR, J. F. ; GOIS, G.; CUNHA-ZERI, G.; ZERI, M. Rainfall variability over Alagoas under the influences of SST anomalies. Meteorology and Atmospheric Physics, Vienna, v. 129, n. 2, p. 157 $171,2017$.

LU, Y.; COOPS, N. C.; WANG, T.; WANG, G. A Process-Based Approach to Estimate Chinese Fir (Cunninghamia lanceolata) Distribution and Productivity in Southern China under Climate Change. Forests, Basileia, v. 6, n.3, p. $360-379,2015$.

PINKARD, E. A.; BATTAGLIA, M. Using hybrid models to develop silvicultural prescriptions for Eucalyptus nitens. Forest Ecology and Management, Amsterdam, v. 154, n. 1-2, p. 337-345, 2001.

STAPE, J. L.; BINKLEY, D.; RYAN, M. G.; FONSECA, S.; LOOS, R. A.; TAKAHASHI, E. N.; SILVA, C. R.; SILVA. S. R.; HAKAMADA, R. E.; FERREIRA, J. M. A.; LIMA, M. N.; GAVA, J. L.; LEITE, F. P.; ANDRADE, H. B.; ALVES, J. M. SILVA, G. G. C.; AZEVEDO, M. R. The Brazil Eucalyptus Potential Productivity Project: Influence of water, nutrients and stand uniformity on wood production. Forest Ecology and Management, Amsterdam, v. 259, n. 9, p. 1684-1694, 2010.

SANDS, P. J.; LANDSBERG, J. J. Parameterisation of 3-PG for plantation grown Eucalyptus globulus. Forest Ecology and Management, Amsterdam, v. 163, n. 2-3, p. 273-292, 2002.

STAPE, J. L.; BINKLEY, D.; RYAN, M. G. Eucalyptus production and the supply, use and efficiency of use of water, light and nitrogen across a geographic gradient in Brazil. Forest Ecology and Management, Amsterdam, v. 193, n. 1-2, p. 17-31, 2004.

SOUZA, J. H.; RIBEIRO, A.; LEITE, H. G.; LEITE, F. P.; MINUZZI, R. B. Disponibilidade hídrica do solo e produtividade do eucalipto em três regiões da Bacia do Rio Doce. Revista Árvore, Viçosa, v. 30, n. 3, p. 399410, 2006.

OLIVEIRA JÚNIOR, J. F.; DELGADO, R. C.; GOIS, G.; LANNES, A.; DIAS, F. O.; SOUZA, J. C. S.; SOUZA, M. Análise da Precipitação e sua Relação com Sistemas Meteorológicos em Seropédica, Rio de Janeiro. Floresta e Ambiente, Seropédica, v. 21, n. 2, p. 140-149, 2014.

WARING, R. H.; COOPS, N. C.; MATHYS, A.; HILKER, T.; LATTA, G. Process-Based Modeling to Assess the Effects of Recent Climatic Variation on Site Productivity and Forest Function across Western North America. Forests, Basileia, v. 5, n. 3, p. 518-534, 2014.

ZHAO, M.; XIANG, W.; PENG, C.; TIAN, D. Simulating age-related changes in carbon storage and allocation in a Chinese fir plantation growing in southern China using the 3-PG model. Forest Ecology and Management, Amsterdam, v. 257, n. 6, p. 1520-1531, 2009.

Recebido em 27/04/2016

Aceito para publicação em 28/03/2017 